Lepr Rev (1992) 63, Supplement, 84s-92s

\title{
The measurement of the epidemiological impact of multidrug therapy
}

\author{
CHAROON PIRAYAVARAPORN \& \\ SOMCHAI PEERAPAKORN
}

One of the well-known effects of multidrug therapy (MDT) when using a mass programme approach is the reduction of prevalence because of the shortened duration of treatment. It is still not known if the application of MDT helps to accelerate the cessation of leprosy transmission in the community, which is the major concern of any leprosy control activities, and be quantified in terms of incidence reduction. This paper is not aimed at giving the unequivocal answer to the question but it presents the current changes in the observed pattern of leprosy in Thailand in relation to the MDT implementation of our leprosy control programme.

\section{MDT and the leprosy control programme in Thailand}

Modern leprosy control programmes based on case-finding and domiciliary treatment was established in Thailand in 1955 as a specialized programme. At that time dapsone monotherapy was the only standard treatment available. The revision of the programme approach from a specialized programme to an integrated programme of the basic health service system was fully effective in 1977, covering 67 of the 73 provinces of Thailand. In the remaining 6 highly endemic provinces, Mahasarakham, Kalasin, Roi-et, Surin, Saraburi, and Nakhon Sawan, leprosy control activities have been maintained and directed using a specialized programme approach by leprosy zonal centres ever since.

MDT implementation was started in 1984 in 3 northeastern provinces (Mahasarakham, Kalasin and Roi-et) which are among the specialized programme areas as mentioned above. From the implementation of MDT it took 7 years to gradually increase the coverage rate among all leprosy patients in Thailand from 11 to $94 \%$ in 1990 (Table 1).

Such an achievement in MDT implementation has been made possible by active and sincere support from many non-governmental organizations (NGOs), since the allocated government annual budget for leprosy control activities alone could not meet the enormous expenses of the MDT implementation programme. For instance, the supply of rifampicin and clofazimine have been adequate because of timely donations from the World Health Organization (WHO) and the Sasakawa Memorial Health Foundation (SMHF), which were in addition to the purchase of drugs from the Government budget. Some other contributions which were also equally important are cars, microscopes, health personnel training expenses, etc. These have been granted by the German Leprosy 
Table 1. Cumulative MDT coverage by year. Thailand, 1984-1990

\begin{tabular}{ccrrr}
\hline Year & \multicolumn{3}{c}{$\begin{array}{c}\text { Province covered } \\
\text { No. }\end{array}$} & \multicolumn{2}{c}{ Case covered } \\
& & \multicolumn{1}{c}{ No. } & $\%$ \\
\hline 1984 & $3^{*}$ & $(4)$ & 5,008 & $(11)$ \\
1985 & 16 & $(22)$ & 13,673 & $(33)$ \\
1986 & 29 & $(40)$ & 18,901 & $(55)$ \\
1987 & 49 & $(67)$ & 18,075 & $(63)$ \\
1988 & 61 & $(83)$ & 14,605 & $(65)$ \\
1989 & 73 & $(100)$ & 13,695 & $(82)$ \\
1990 & 73 & $(100)$ & 11,308 & $(94)$ \\
\hline
\end{tabular}

* Mahasarakham, Kalasin, Roi-et/Northeast of Thailand.

Relief Association/Hartdegen Fund (GLRA/HF), The Netherlands Leprosy Relief Association (NSL), the American Leprosy Mission (ALM), and innumerable other domestic and international NGOs as well.

Case-finding methods in Thailand consist of household contact surveillance, rapid village surveys (mobile clinic), school surveys, and skin clinic services. All methods have been practised regularly since the vertical programme of 1955 with various degrees of intensification in some methods during that time. Figure 1 shows the annual achievement in terms of the number of persons examined from 1976 to 1990.

\section{Changes in the observed pattern of leprosy: a nationwide comparison and the findings of 3 provinces}

The data mentioned here are from routine recording and reporting of all governmental health service units which are parts of the leprosy control programme. Data are collected nationwide from all 73 provincial health offices, 12 leprosy zonal centres, and 13 government-run leprosy settlements. Data from the 3 provinces, Mahasarakham, Kalasin and Roi-et, are grouped as one unit to make comparison with the nationwide data more meaningful.

The years 1984-1990 should be considered as a transition period during which MDT gradually replaced dapsone monotherapy in Thailand (as mentioned above) on a nationwide scale. Hence it may be too early to claim, in 1991, that any change in the occurrence of new cases as represented by the nationwide leprosy detection rate is due to MDT. On the contrary, for the 3 provinces where MDT coverage of leprosy cases was high from the start and exceeded $95 \%$ within 1 year of operation owing to the vertical programme settings, any change in the new case profile there could possibly be originated from MDT implementation. In addition, even though the leprosy control programme has been operating for some time in Thailand without major changes in case-finding

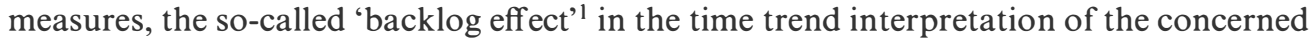
rate should still be kept in mind.

Changes in the observed pattern of leprosy are as follows: 


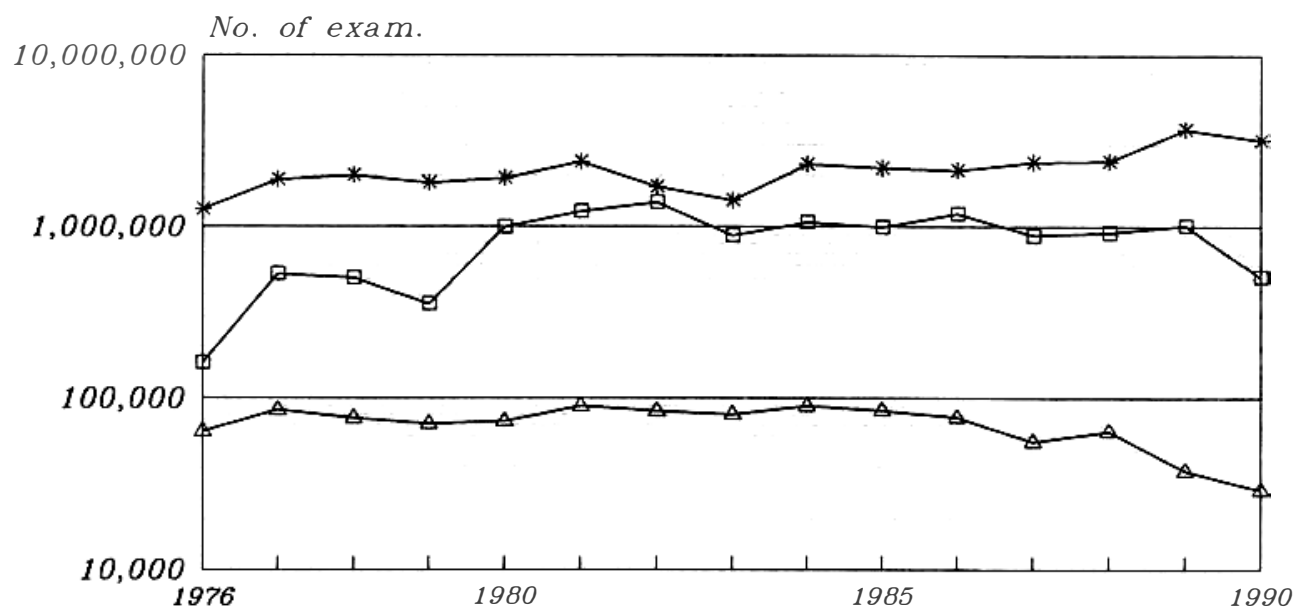

Figure 1. Leprosy case-finding activities, Thailand, 1976-90. $\triangle$ Household contact, $\square-$ Mobile clinic, $\rightarrow$ School survey.

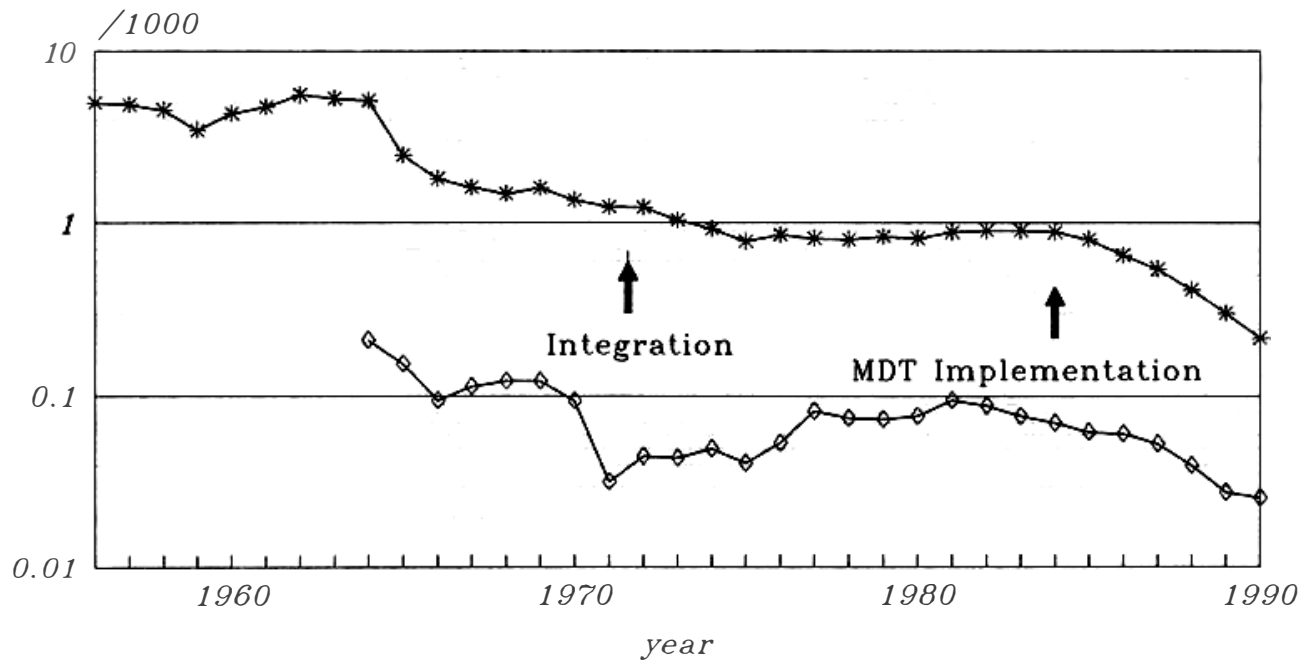

Figure 2. Leprosy in Thailand, 1956-90. $\nleftarrow$ Prevalence rate, $\diamond$ Detection rate.

\subsection{Prevalence and detection rate reduction (Figures 2-5)}

Since the beginning of the integration programme in 1976, a marked prevalence rate reduction was observed after the implementation of MDT in 1984. It is observed both on a nationwide scale and in the 3 provinces with, not surprisingly, a greater rate of decline in the latter due to the said historical background of MDT implementation.

An interesting finding is the regular decline of the detection rate which showed up before MDT. The detection rate decline in Thailand began in 1982 and in 1983 in the 3 provinces, which is definitely before MDT could have had any effect. Additionally, the 


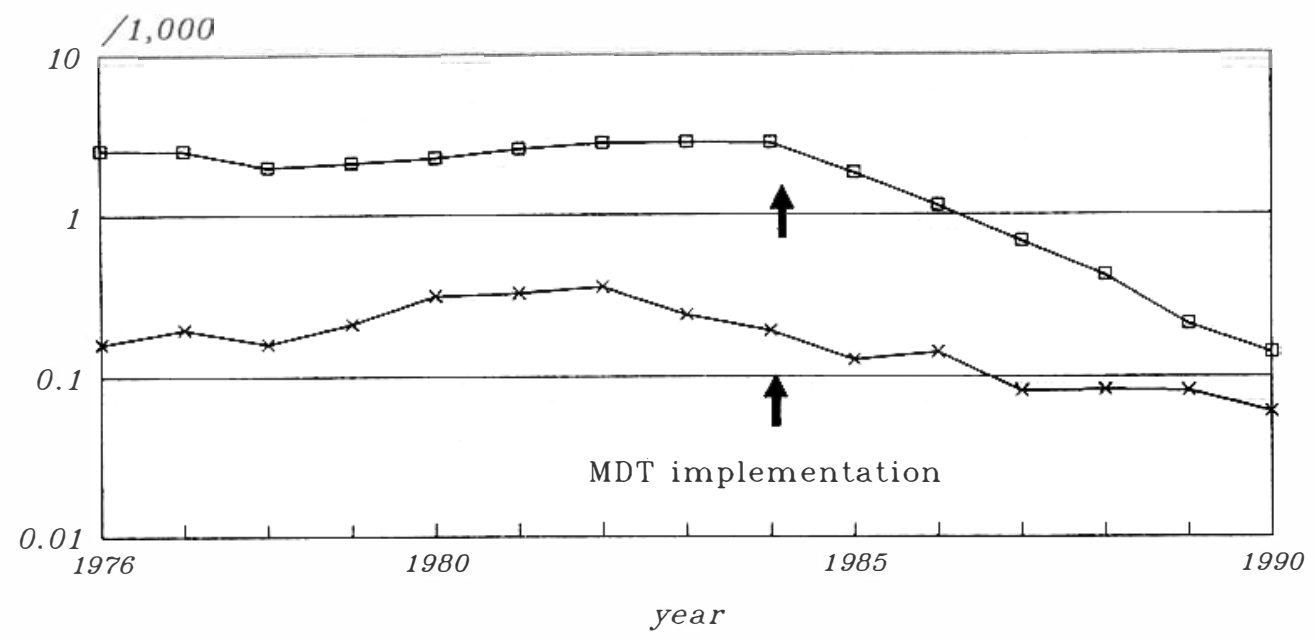

Figure 3. Leprosy in 3 provinces, Mahasarakham, Kalasin and Roi-et, of North-east Thailand, 1976-90. $\square-$ Prevalence rate, $\rightarrow$ Detection rate.

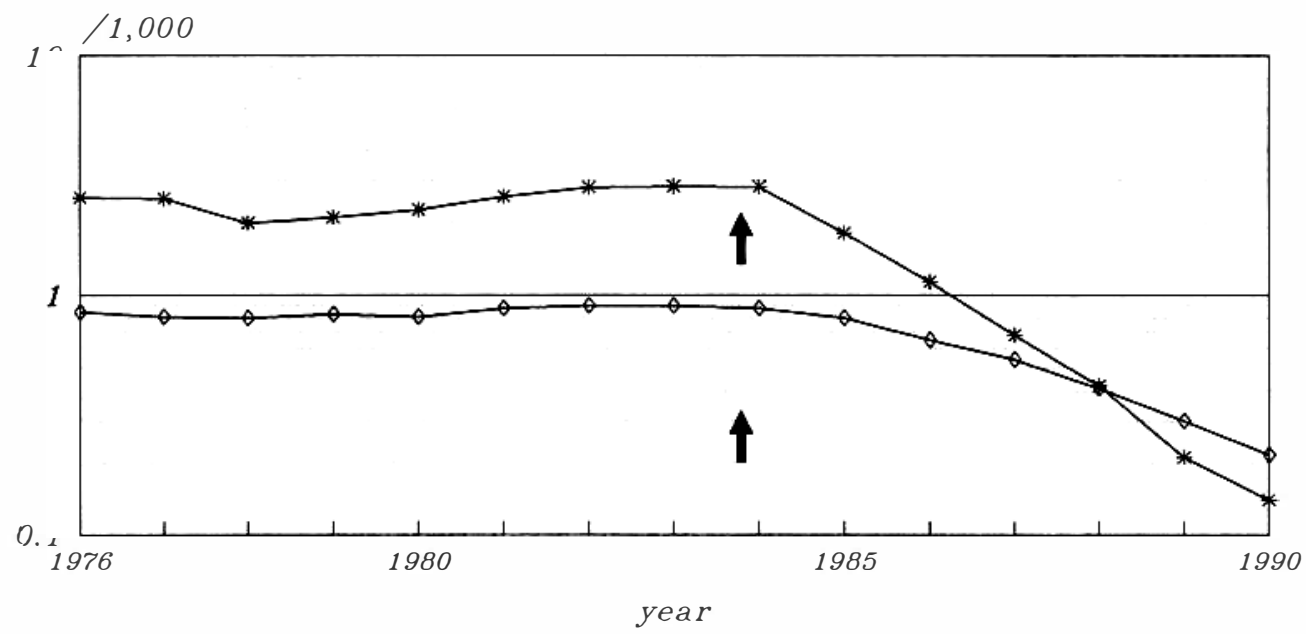

Figure 4. Leprosy prevalence rate in all provinces vs 3 provinces in Thailand, 1976-90. $\longleftarrow 3$ provinces, $-\diamond$ All provinces.

falling rate of the detection of leprosy is greater in the 3 provinces than in Thailand. Anyhow, the decline still continued after the introduction of MDT.

Theoretically, the prevalence to detection ratio under a MDT programme should be less than the ratio under a dapsone monotherapy programme due to the shortened period of treatment. This theory has been confirmed since the ratio in the 3 provinces has reduced from 14.7 in 1984 to 2.4 in 1990 , whereas in the case of Thailand the ratio was 12.5 in 1984 and 8.4 in 1990 . 


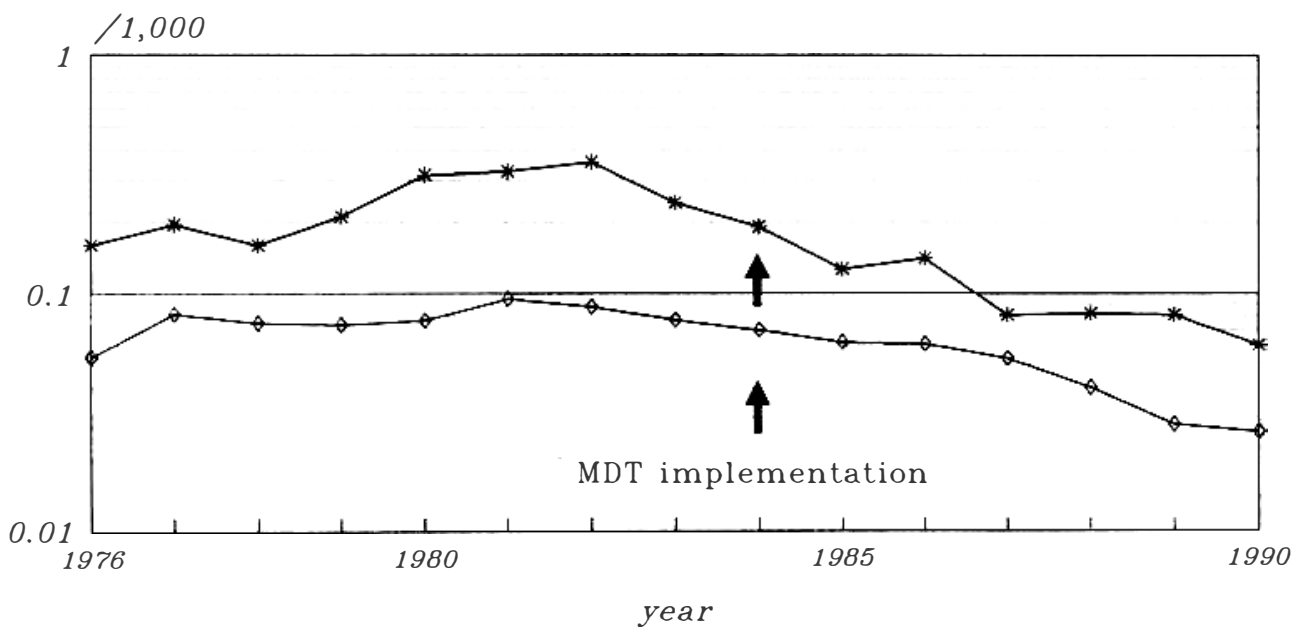

Figure 5. Leprosy detection rates in all provinces vs 3 provinces in Thailand, 1976-90. $\nleftarrow 3$ provinces, $\diamond$ All provinces.

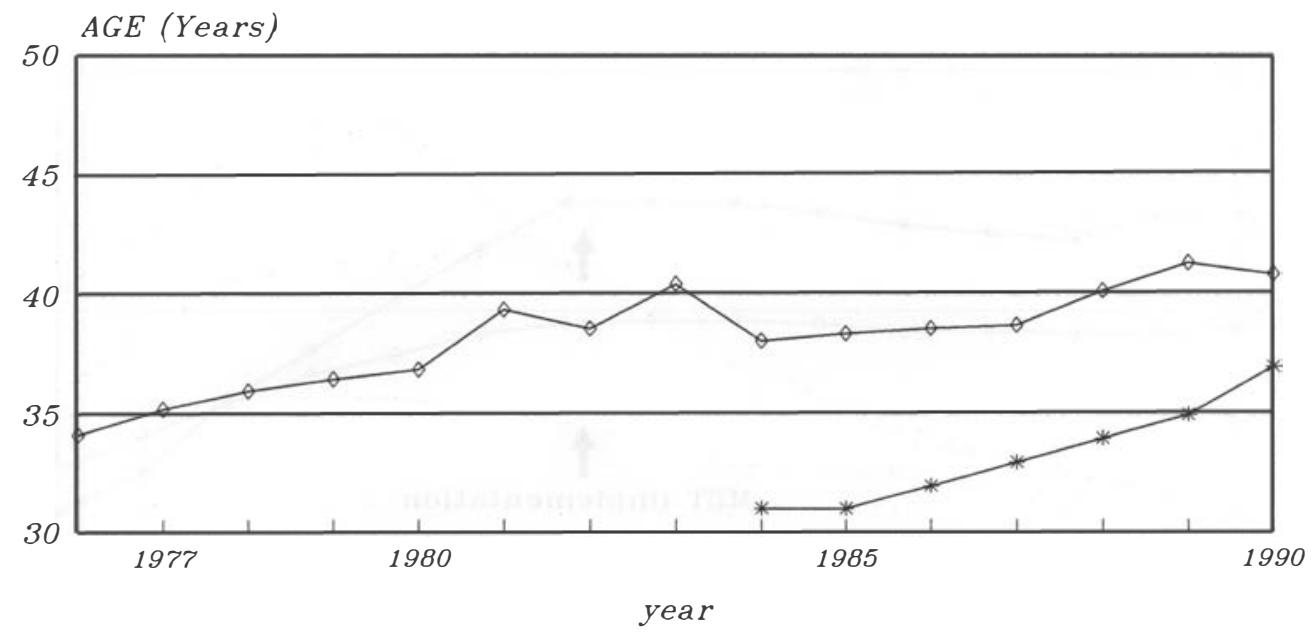

Figure 6. Mean age at onset among new cases of leprosy, Thailand, 1976-90. $\diamond$ All provinces, $\rightarrow-3$ provinces.

\subsection{An increase in mean age at onset and a shift in the peak age incidence (Figures 6-10)}

There is a gradual increase in the mean age at onset among new leprosy cases in Thailand from $34 \cdot 16$ years in 1976 to $40 \cdot 87$ years in 1990 and in the 3 provinces from 31 years in 1984 to 37 years in 1990 .

Analysis of the age-specific leprosy detection rate in Thailand and the 3 provinces using 5-year average data during 1976-1990 reveals a similar pattern. There is a general decline in the detection rate in every age group during that period. The peak age of 


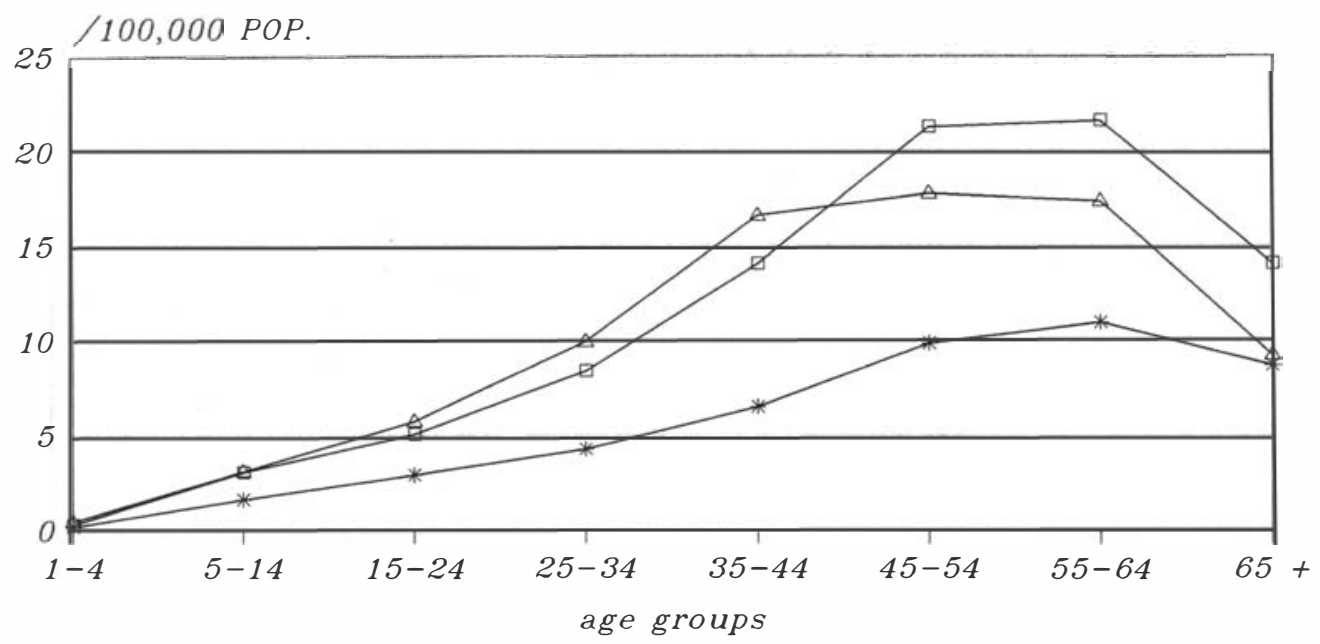

Figure 7. Age specific annual detection rate of leprosy, Thailand, 1976-90. (MDT started in 1984 and)- $\Delta-$ Year 1976-80, - - Year 1981-5, ※ Year 1986-90.

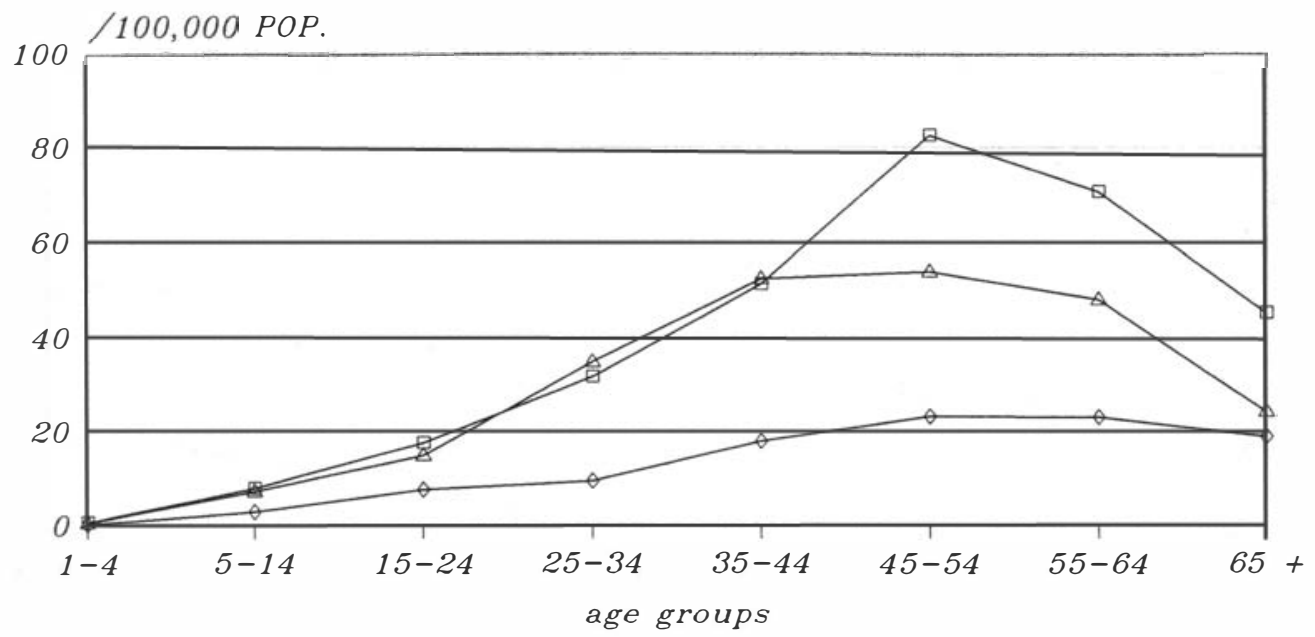

Figure 8. Age specific annual detection rate of leprosy, 3 provinces in Thailand, 1976-90. (MDT started in 1984.) $\triangle$ Year 1976-80, $\square-$ Year 1981-5, $\diamond$ Year 1986-90.

incidence is in the older adult population in Thailand and also in the 3 provinces. A shift in peak age incidence to the older age group is clearly observed in Thailand and in the 3 provinces as well by comparing the data of 1980, 1985 and 1990.

\subsection{An increase in the lepromatous ratio among new cases (Figure 11)}

A clear, steady increase of the lepromatous ratio among new leprosy cases has been observed both in Thailand and in the 3 provinces after the implementation of MDT. In 


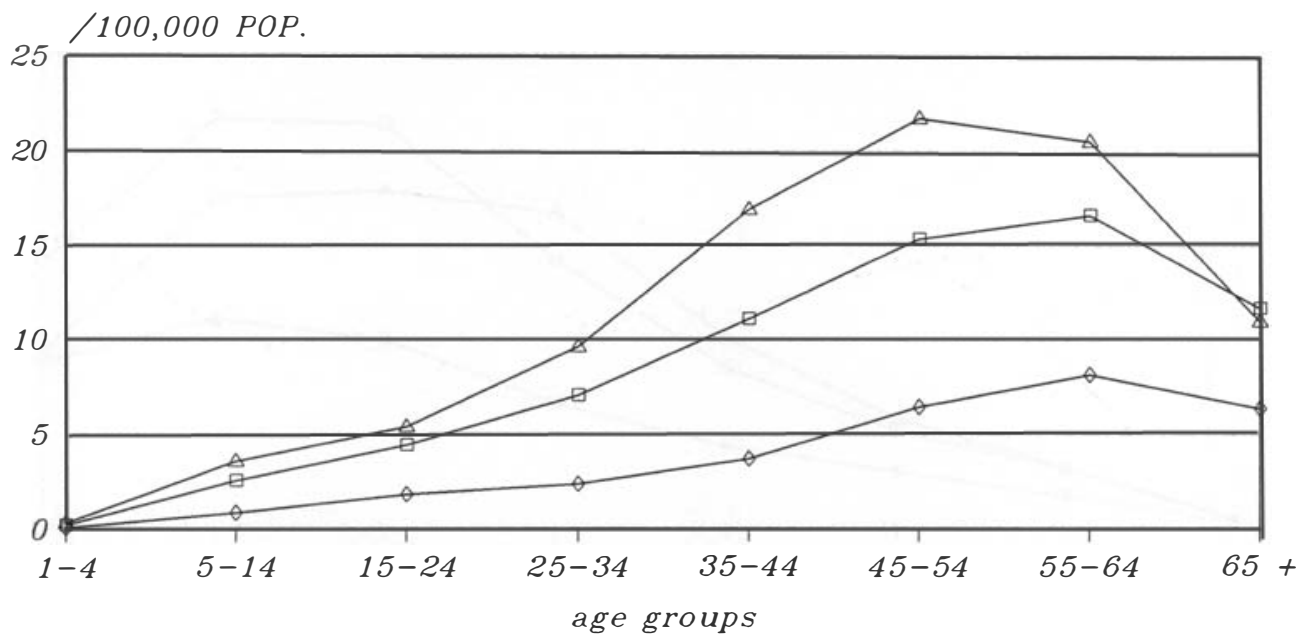

Figure 9. Leprosy detection rate by age group, Thailand, 1980, 1985 and 1990. $\triangle$ Year 1980, $\square-$ Year 1985, $\diamond$ Year 1990 .

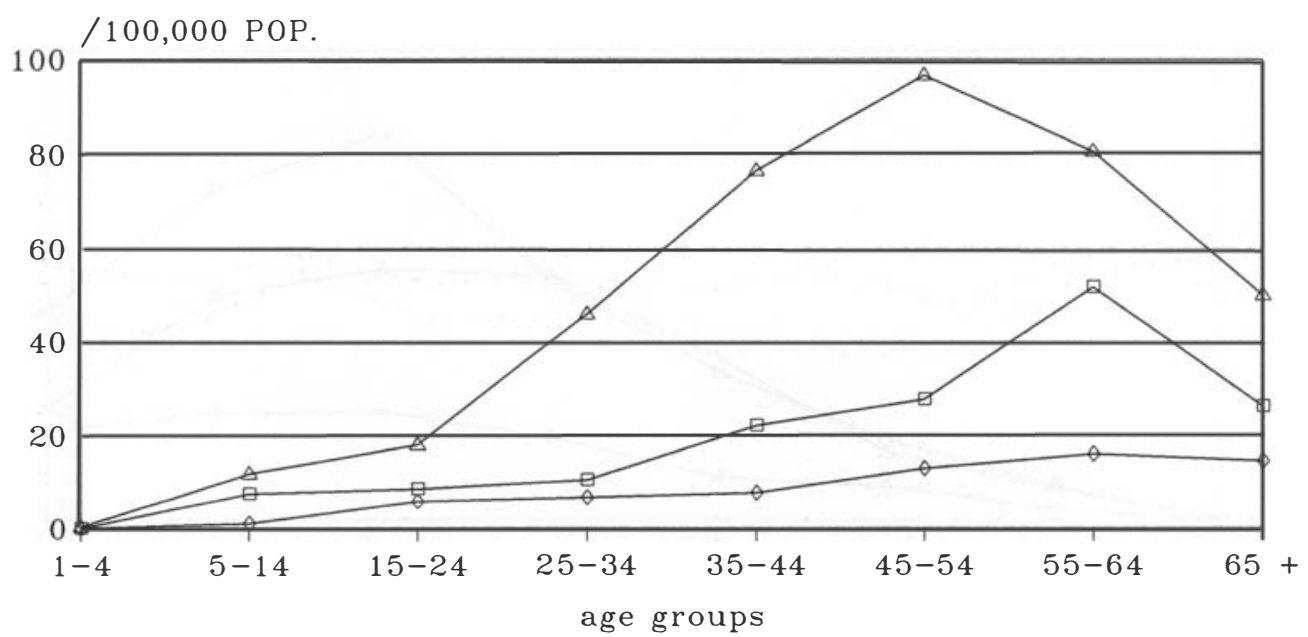

Figure 10. Leprosy detection rate by age group, 3 provinces (Mahasarakham, Kalasin and Roi-et), Thailand, 1980, 1985 and 1990. $\triangle$ Year 1980, $\square$ Year 1985, $\diamond$ Year 1990.

the case of Thailand the increase has been for a long while and in the 3 provinces it only began in 1985 .

The lepromatous ratio in the 3 provinces, together with the average age-specific detection rate pattern of the 'pre-MDT' period (1981-1985) has reminded us of the 'backlog effect' possibility. ${ }^{1}$

\subsection{A steady trend of disability rate among new cases (Figure 12)}

The rate of leprosy disability (grade 2 and over) among new cases is lower in the 3 


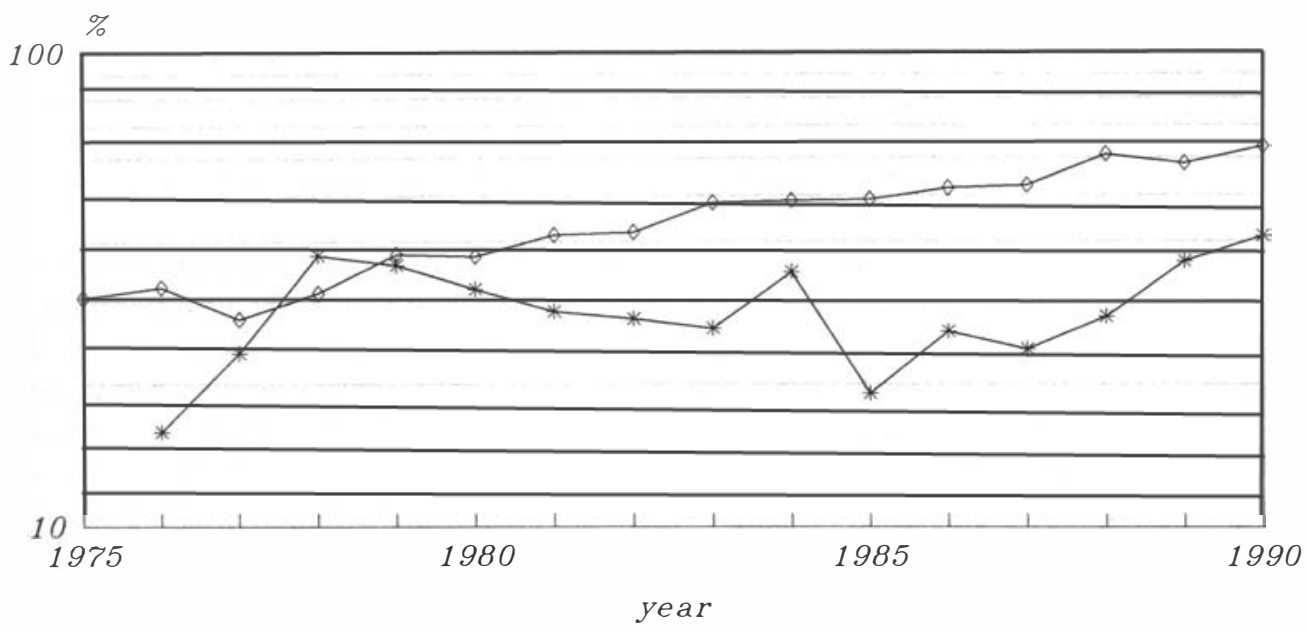

Figure 11. The lepromatous ratio among new cases of leprosy, Thailand, 1975-90. $\diamond$ All provinces, $\rightarrow 3$ provinces.

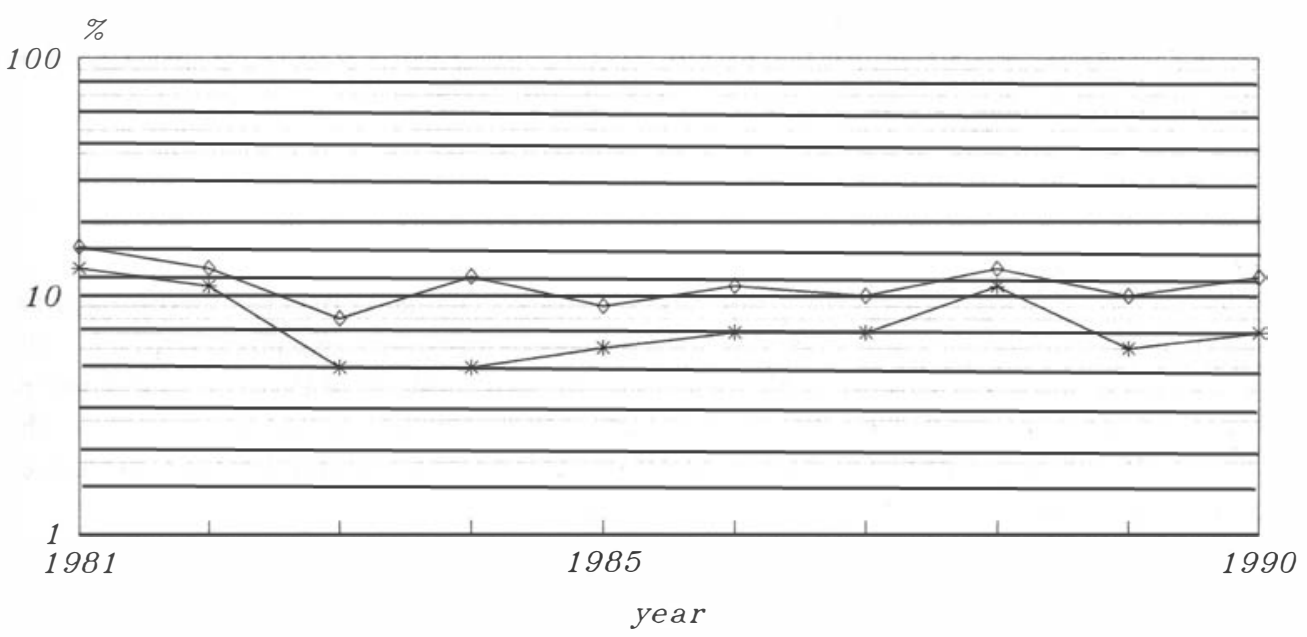

Figure 12. The disability rate among new cases of leprosy, Thailand, 1981-90. $\diamond$ All provinces. $\rightarrow 3$ provinces.

provinces than in the nationwide data implying earlier case detection. Due to the laborious effort in case detection activities under the vertical programmes of the 3 provinces, a single figure disability rate has been achieved and quite successfully sustained during the period.

\section{Conclusion and Discussion}

Today Thailand can be regarded as one of the low endemicity areas of leprosy, because of 
the low prevalence and detection rates. The secular trends of major epidemiological indicators such as the increase of the mean age at onset, the increase of the lepromatous ratio among new cases, and the shift to the older age group of the peak age of incidence, all of which are quite definite, imply that at present the occurrence of leprosy is the result of post-primary infection of earlier days and that leprosy transmission in Thailand is now inactive.

Since the case detection rate serves as an estimate of incidence and is greatly influenced by the type and intensity of case-detection activities, ${ }^{2}$ the validity of the detection rate as an indicator of incidence should be assessed first. According to Lechat, ${ }^{3}$ when the proportion of lepromatous subjects become constant and when the proportion of new patients with disabilities approaches zero, the detection rates can be considered as a valid estimation of the incidence rates. Based on these criteria, the decline in the detection rate in our study could not be validified as the decline in leprosy incidence since the L-ratio is not constant and the disability rate among new cases is not approaching zero. However, the said findings in the 3 provinces, together with the subjective impression of the leprosy control team there, convinced us that the said criteria may not be practical or sensitive enough for the situation of low endemicity in our case.

Should a new set of epidemiological indicators be identified regarding the assessment of active leprosy transmission in the community of low leprosy endemicity?

Should there be some studies trying to make use of modern technology in the field of seroepidemiology and immunology to assess the dynamics of leprosy transmission as supplementary data to the study of epidemiological indicators?

\section{Acknowledgments}

The authors would like to thank Mr Sinchai Kachend of the Leprosy Division and Dr Yongyuth Wangroongsarb of the Malaria Division for their excellent work on the graphic presentation in this article. We are also indebted to Mr Piya Piyasilp, the Director of the Leprosy Zonal Centre, 6 Khon Kaen and Dr Pieter A M Schreuder of the Netherlands Leprosy Relief Association (NSL) for their contributions on the 'three provinces' data set.

\section{References}

1 World Health Organization. Epidemiology of leprosy in relation to control. Technical Report Series 716, Geneva, 1985: 34-37.

2 World Health Organization. Epidemiology of leprosy in relation to control. Technical Report Series 716, Geneva, 1985: 33.

3 Lechat MF. Control programs in leprosy. In Hastings RC. Ed. Leprosy. Churchill Livingstone, Hong Kong, 1985: 264. 